\title{
Protée
}

\section{Comment faire apparaître Écho ? Soeurs, saintes et sibylles de Nan Goldin et Autoportrait en vert de Marie Ndiaye}

\section{Martine Delvaux et Jamie Herd}

Volume 35, numéro 1, printemps 2007

Échos et résonances

URI : https://id.erudit.org/iderudit/015886ar

DOI : https://doi.org/10.7202/015886ar

Aller au sommaire du numéro

\section{Éditeur(s)}

Département des arts et lettres - Université du Québec à Chicoutimi

ISSN

0300-3523 (imprimé)

1708-2307 (numérique)

Découvrir la revue

Citer cet article

Delvaux, M. \& Herd, J. (2007). Comment faire apparaître Écho ? Soeurs, saintes et sibylles de Nan Goldin et Autoportrait en vert de Marie Ndiaye. Protée, 35(1), 29-39. https://doi.org/10.7202/015886ar

\section{Résumé de l'article}

Cet article étudie l'usage du portrait photo-textuel dans l'écriture autobiographique au féminin, par le biais de l'analyse parallèle de Soeurs, saintes et sibylles de Nan Goldin, et de Autoportrait en vert de Marie Ndiaye. Grâce au jeu entre photographie et littérature, ces objets intermédiatiques présentent une structure en écho (et reposent sur une série d'échos) qui permet de revoir le mythe d'Écho et de Narcisse ainsi que le rapport entre le visuel et le verbal. Goldin et Ndiaye fondent leur travail sur l'indécidabilité de la photographie pour écrire entre le mythe et l'histoire personnelle, le sacré et le profane. Par ailleurs, les oeuvres étudiées peuvent être lues comme des légendes et des échos dans le cadre du corpus de chacune des auteures. Ces pratiques intermédiaires mettent en place une série de résonances qui permettent aux femmes, à l'intérieur des deux textes, d'être vues et entendues. Par le vacillement entre portrait et autoportrait, ces pratiques réussissent à faire apparaître des visages là où il y avait des voix. 


\title{
CO M M ENT FAIRE APPARAÎTRE ÉCHO? SEEURS, SAINTES ET SIBYLLES DE NAN GOLDIN ET AUTOPO RTRAIT EN VERT DE M ARIE N DIAYE
}

\author{
MARTINE D ELVAuX ET JAM IE HERD
}

L'œuvre contemporaine est-elle le lieu, singulier, nouveau, où peuvent se rencontrer les voix, où les voix peuvent se faire écho, devenir des échos? Un nombre remarquable d'œuvres intermédiales, produites par des femmes, ont fait leur apparition sur les scènes littéraire et artistique au cours de la dernière décennie. Quel est ce rapport particulier qu'ont les écrivaines et photographes à l'usage conjoint des mots et des images photos? Comment cette pratique féminine se rapproche-t-elle d'un "bon usage" de l'écho qui se distingue d'une historicisation, d'un travail de l'archive - bibliothèque de soi ou album de famille - plus aisément identifiable dans le cas d'œuvres masculines?

Aux fins de cette réflexion, nous avons choisi non seulement de nous pencher sur deux artistes et deux œuvres dont le fonctionnement interne est en écho, mais aussi de les mettre en écho, de les penser en côte-à-côte, dans leur réverbération et leur réflexion. D'une part, une photographe, Nan Goldin, qui, dans son livre essentiellement photographique Soeurs, saintes et sibylles (2005), fait une incursion dans le domaine littéraire, entre autres par le biais de la citation; d'autre part, Marie Ndiaye qui, dans Autoportrait en vert (2005), un livre essentiellement littéraire, fait appel à l'image photographique par l'entremise de clichés anciens et d'une collaboration avec la photographe Julie Ganzin. Dans les deux cas, on trouve un travail avec l'autre - l'autre médium et l'autre voix - et une approche de thèmes communs aux deux artistes, thèmes qui tournent autour du féminin, de cette figure invisible, inimaginable, en somme infigurable, qu'est la femme. La sœur chez Goldin, la mère chez Ndiaye sont le point d'ancrage d'un travail de l'apparition: comment faire apparaître la femme, comment la voir, comment figurer sa voix?

Si Narcisse refuse de regarder Écho, de répondre à son appel et de voir son visage, Goldin et Ndiaye, par le jeu intermédial entre le mot et l'image, le sonore et le visuel, jeu que redoublent l'énonciation et l'imagination des ouvrages, viennent conférer à cette voix un visage. Sous leur plume et dans leur objectif, Narcisse est forcé de voir Écho, et Écho retrouve ainsi un certain narcissisme.

Les ouvrages choisis, Sœurs, saintes et sibylles et Autoportrait en vert, pourraient être décrits, dans le cas de Goldin comme dans celui de Ndiaye, comme un ersatz 
et aussi une mise en abyme de l'œuvre. Incursions avouées dans l'autobiographique - le livre/exposition/ performance ${ }^{1}$ de Goldin est un témoignage au suicide de sa sœur aînée, événement fondateur dans sa pratique artistique; l'autoportrait de Ndiaye repose sur une série de portraits de femmes en vert que l'auteure rassemble pour refléter les contours d'un autoportrait-fictif -, ces productions singulières n'en sont pas moins le lieu d'un jeu formel qui, d'une part, crée des effets de bruitage qui brouillent la limpidité et le "gage» de sincérité associés au «je», et qui, d'autre part, en tant que microcosme ou mise en abyme des œuvres respectives, fait apparaître un savant réseau d'échos (citation, reprise, reproduction, intertextualité....). Ainsi, Soeurs, saintes et sibylles et Autoportrait en vert sont des substrats: non seulement un espace clos à l'intérieur duquel «ça» résonne, à force de répétitions et de redoublements, mais un centre où, aussitôt créé et projeté, le son vient se répercuter contre des productions antérieures, le nouveau rappelant l'ancien, l'ancien se trouvant transformé par son visage nouveau. On entend l'écho, le son revient mais il n'est plus comme avant : quelque chose d'autre apparaît, le même est différent. Dans l'objectif de Goldin et sous la plume de Ndiaye sont rejoués les éléments du mythe, la voix et le miroir de l'eau. La démultiplication de la figure féminine qui s'opère par l'emploi du portrait, le brouillage de son propre visage sur lequel l'artiste se penche comme s'il se trouvait au-dessus de l'eau, le retour des voix comme des revenantes, fantômes oubliés sur qui les auteures jettent la lumière: voilà comment se décline ici le travail de l'écho. Aussi différentes soient-elles, la photographe américaine Nan Goldin et l'écrivaine française Marie Ndiaye se rejoignent dans l'utilisation intermédiale et autobiographique du récit et de la photographie, croisement et face-à-face qui empêchent l'identification générique des œuvres, comme celle des individus qu'elles sont censées représenter. Épris de son image, Narcisse a trouvé la mort dans l'eau, abandonnant Écho comme une veuve avortée. Goldin et Ndiaye échappent à cet amour de soi mortifère, choisissant l'image, la voix et leurs multiples visages, jouant de la biographie comme d'un pavé jeté dans une mare, qui laisse derrière lui son passage, en cercles concentriques. Le suicide de Narcisse est vain, et Écho survit.

Walter Benjamin décrivait l'aura comme «une trame singulière d'espace et de temps: l'unique apparition d'un lointain, si proche soit-il»(2003: 19). Le drame que Benjamin associait à l'époque de la reproductibilité technique concernait le désir humain de "posséder l'objet d'aussi près que possible, dans l'image ou, plutôt, dans son reflet» (ibid.: 20-21). Ce désir de possession contraste avec la production de l'œuvre d'art unique qui reposait sur une tradition, un rituel, en somme un culte. Avec la sécularisation de l'art, c'est l'authenticité qui vient se substituer au culte ${ }^{2}$. Mais comment parler d'authenticité en matière de photographie? Comment penser la photographie sinon comme un jeu d'échos?

Le critique Philippe Dubois associe la photographie à un principe de distance irréductible et fondamental distance entre le réel et l'image, entre l'objet saisi et le photographe, enfin entre le sujet qui regarde la photo et l'objet représenté. Cette distance correspond à l'effet auratique benjaminien, dialectique issue de la tension entre le lointain et le proche, le "lointain le plus essentiel tenu, maintenu, dans le proche le plus événementiel» (Benjamin, 2003: 31). Selon Georges Didi-Huberman, c'est en sécularisant l'aura de Benjamin qu'on peut comprendre et rendre compte de l'efficacité étrange et unique de nombreuses œuvres modernes qui déconstituent les valeurs cultuelles, les cultures déjà informées. Les artistes comme les enfants, explique DidiHuberman, montrent que la distance n'est pas l'apanage du divin; si la religion constitue le paradigme historique et la forme anthropologique exemplaire de l'aura, ce n'est pas la seule façon de comprendre cette dernière. De fait, c'est bien la modernité qui a permis de briser ce lien et d'ouvrir un rapport qui était clos. Là, l'aura devient le sublime, la forme pure de ce qui surgit; dès lors, comme l'écrit Didi-Huberman, l'art moderne se fait le lieu où 
quelque chose nous parle de la hantise "comme de ce qui nous reviendrait de loin, nous concernerait, nous regarderait et nous échapperait tout à la fois» (1992: 104).

Le portrait, pour Benjamin, demeurait le dernier bastion de l'aura, mais qu'en est-il de l'autoportrait? Si on peut dire que sous la couche du visage pris sur la photo se cache une série de visages ("de qui au juste est un visage photographié?», demande Philippe Lacoue-Labarthe [1979: 24]), on doit aussi affirmer qu'il n'y a pas de visage de soi sinon comme ce qui toujours échappe au sujet qui (se) voit. Le visage dévisage si on croit, comme Jacques Cohen, que l'action de dévisager consiste à chercher le visage dans le langage et dans l'image, l'expression dans la représentation. Car pour Cohen, comme pour Didi-Huberman, ce visage regardé avec intensité est aussi un point de non-voir, point de fuite où le regard s'engouffre. L'effet de focalisation médusante des visages fonde ainsi leur effet de métamorphose et, à la fin, c'est comme hantise que le visage se révèle.

\section{I'll be your mirror}

Pour Benjamin, l'aura survivait dans le portrait photographique où le visage humain représentait l'ultime retranchement de la valeur cultuelle (qu'il trouvait dans l'expression fugitive d'un visage ou le culte du souvenir des disparus [2003: 32]). On pourrait dire du travail de Nan Goldin qu'il fait le grand écart entre l'auratique du portrait et la reproductibilité séculaire, pour faire naître une œuvre qui se déploie entre le religieux (ou le mythique) et le séculier, œuvre qui en somme surgit de leur rencontre, constituant une approche de ce qu'on pourrait nommer un désacré, un sacré décultualisé, un sacré bien moderne mais néanmoins sacré ${ }^{3}$. Ce sacré-qui-n'en-est-pas-un apparaît dans l'objectif de la photographe, par le regard qu'elle pose sur les sujets choisis, ce regard non pas voyeur mais avant tout empathique et mélancolique, créateur de communauté:

There is a popular notion that the photographer is by nature a voyeur, the last one invited to the party. But I'm not crashing; this is my party. This is my family, my history. 4

Ainsi, les milliers d'instantanés pris par Goldin au fil des ans renvoient les uns aux autres, fils invisibles reliant les individus, à travers l'espace et le temps. C'est là la "famille de Nan ${ }^{5}$, cette communauté désœuvrée dont elle fait partie, communauté d'amis et d'amants qui se défait et se refait constamment, ce que traduisent les expositions, catalogues et

\section{Est-elle une femme en vert?}

Jean-Marie Schaeffer écrit:

Pour peu qu'on s'interroge sur le statut réel du portrait

photographique, on découvre que, loin de célébrer l'autonomie

d'un "moi» se mirant dans sa propre autosuffisance, il ne cesse

de nous renvoyer à notre absence de plénitude. (1997: 21)

Le portrait photographique n'appartient pas, selon Schaeffer, au culte moderne de la subjectivité autonome, mais donne la preuve que la subjectivité dépend de l'interaction avec autrui dont elle est constituée. C'est un jeu de regards qui laisse une trace, une image du passé absent et, dans l'écart du temps qui caractérise la prise photographique, «[l]e "moi" est toujours déjà devenu un autre" (ibid.: 23). Le portrait photographique est donc paradoxal puisque, même s'il y a distance entre photographe et sujet photographié, le portrait promet l'accès à un sujet perçu, du moins en partie, comme un objet que le photographe peut saisir. Ainsi, le portrait a un statut impur (ibid.: 16): une même photo peut être qualifiée aussi bien d'objet artistique que de preuve d'identité juridique.

L'écriture de Marie Ndiaye est constituée des contradictions soulevées par Schaeffer. Sa fiction interroge l'identité et la montre comme une chose instable et changeante, qui naît de l'interaction avec les autres, surtout dans le rapport familial. Les romans En famille et Rosie Carpe proposent des 
diaporamas dont la forme n'est jamais arrêtée. C'est un mouvement de marée que Goldin capte, la caméra à la main, comme si celle-ci était une extension de son corps, photographiant à répétition ceux qui font partie de sa vie. Une façon de les toucher, de les caresser; une façon, aussi, de faire résonner la voix de ceux qu'on refuse d'entendre parler, qu'on nomme le plus souvent pour les exclure - les travestis, les transsexuels, les toxicomanes, les démunis, mais aussi, toujours, les femmes - , la parole servant à les cristalliser en stéréotypes ou en symptômes (Lebovici, 1999), en des icônes, des morts-vivants.

Dans son œuvre la plus récente, Sœurs, saintes et sibylles, Goldin effectue un retour sur un événement fondateur, signalé à plusieurs reprises dans son œuvre antérieure (dans des dédicaces, des remerciements ou des préfaces), mais jamais jusque-là confronté et transformé par l'art. Sœurs, saintes et sibylles constitue un témoignage à la sœur aînée de Goldin, Barbara Holly, qui s'est enlevé la vie alors que Nan avait 11 ans. Ce triptyque est un «hommage à ma sœur et à toutes les femmes rebelles qui se battent pour survivre dans la société» (quatrième de couverture), hommage d'abord à Sainte-Barbara, puis à Barbara Holly, et enfin à Nan Goldin elle-même dans un retour sur sa propre vie. Ce livre-hommage, livre-missel, est une chambre de miroirs et une messe des morts ${ }^{6}$.

La figure de la sœur est d'emblée convoquée, cette sœur héroïque, rendue mythique par son suicide, mystifiée par le discours parental et par un contexte social qui, dans les années 1950 et 1960, interdisaient aux filles l'imagination, la sexualité, la liberté. SainteBarbara est un double de cette sœur sacrifiée et sanctifiée par sa cadette. Suivant la légende, prom queen enfermée par son père en haut d'une tour dans le but de préserver sa virginité, elle est devenue sainte - holy - en taillant une troisième fenêtre dans un des murs de sa prison, forme de baptême auto-effectué. Elle a été décapitée par son père, lui-même ensuite foudroyé par un Dieu-justicier. Cette légende chrétienne, morceau de vie des saints, hante le nom de l'enfant juive, comme son destin se trouvait déjà tracé dans l'histoire du petit Jésus. Nan Goldin portraits troublants des personnages, surtout des héroïnes. En famille, en particulier, accorde une place prioritaire au portrait: Fanny vole des clichés de ses tantes dans sa quête d'intégration; cependant, les visages flous de ces portraits ne lui permettent jamais d'authentifier l'identité de l'autre et, le plus souvent, ils embrouillent sa recherche. Autoportrait en vert, publié en 2005, revient sur la question de l'identité en promettant de livrer une image, littéraire ou photographique, de l'écrivaine elle-même. Mais Ndiaye, jalouse de son intimité, ne se livre que par le biais d'une mise en fiction de soi qui passe par les portraits d'autres femmes. De ce fait, son autoportrait rappelle l'impureté du portrait photographique, entre fiction et autobiographie. Dans l'absence de sa propre image, Ndiaye écrit à la manière d'une photographe. Dans les portraits qui parsèment son texte, elle pose son regard sur les femmes qui l'entourent pour faire, par l'écriture, leurs portraits. Son écriture mime l'acte photographique et crée des résonances entre texte et image.

Autoportrait en vert s'ouvre sur le portrait photographique d'une autre - portrait d'une autre femme faite par une autre femme (la photographe Julie Ganzin) - et sur un récit collectif. Cette première photographie montre une femme de dos, aux cheveux blonds, qui se fond dans un paysage flou au dégradé vert. Sur la page opposée, la narration de Ndiaye commence avec une description de la montée des eaux de la Garonne. Bien qu'on discerne, dans l'image, la montagne Sainte-Victoire ainsi qu'une forêt, le flou de la photographie et la position centrale de la figure donnent l'impression qu'il s'agit d'une image de la mer, d'une photographie de baignade, voire de noyade, surtout en regard du texte qui lui est associé. La photo est liée au titre par la couleur, le vert, et finalement à la narration qui, par un glissement, passe du «nous» au «je» de la narratrice écrivaine.

Femmes vêtues de vert, aux yeux verts ou camouflées dans des arbres verts, les femmes de Ndiaye se reflètent les unes dans les autres et se 
annonce la venue de Barbara après sa disparition, la ressuscitant par l'entremise d'un récit-sœur qui lui fait écho - un signe vu après coup comme avant-coureur. La légende de Sainte-Barbara est le cadre de l'histoire, mais c'est aussi le point de fuite qui emporte l'image, la tire en son centre disparu comme dans un trou abyssal. Il faut ainsi lire l'histoire de Sainte-Barbara comme une légende de l'œuvre de Goldin, à la manière de ces phrases inscrites en petits caractères à l'extérieur du cadre d'une photo et dont Goldin se sert pour nommer la singularité de ceux qu'elle photographie et qui, autrement, seraient des personnages et de véritables clichés. L'histoire de Barbara force une lecture à rebours, en échos, de l'œuvre: jusque-là, nulle part elle n'apparaissait, mais les amis, les amants, la famille adoptive, souffrants par la toxicomanie, l'amour ou le sida, dessinaient partout en creux la sœur perdue; un lien sororal qui lie Goldin à ceux qui portent sur eux les traces de la souffrance et de la violence.

C'est d'ailleurs un autre hommage qui se dessine dans les premières pages du livre Sœurs, saintes et sibylles, car la «grid» qui ouvre le volume juxtapositions de photos sous la forme d'une grille présentant la vie de Sainte-Barbara - rappelle les «Positive grids» créées par la photographe pour traiter de la séropositivité et du sida. Ces séries, si elles racontent une histoire (histoires d'amour au temps de la pandémie), si elles créent une narrativité, sont marquées par un jeu du lien et de l'écart. Sorte de romans-photos où les seuls mots qui apparaissent sont ceux d'un titre, où les tableaux s'enchaînent, où une progression est dessinée, le plus souvent un trajet inévitable vers la mort. Ainsi se présente la vie de Barbara, marquée par un destin auquel il était impossible d'échapper, oracle imposé par la suite à Nan elle-même dont on avait affirmé qu'elle finirait comme sa sœur aînée: «Je croyais que je devrais me tuer à 18 ans. Mes parents se mirent à me traiter comme Barbara. À 13 ans je voulais devenir une droguée» (2005: n.p.).

Mais avant la grille initiale de Sainte-Barbara, deux photos (en pages pleines) ouvrent le livre: la première démultiplient à travers la narration. Ndiaye détourne et retravaille certains clichés de la photographe Julie Ganzin; elle ponctue ses mots avec d'autres portraits photographiques visiblement issus d'une époque éloignée (provenant d'une «collection particulière»). Ces images, qui n'illustrent pas directement la narration, fonctionnent comme des citations visuelles; retirées de leur contexte d'origine, elles tracent les contours de la femme en vert, cette figure qui fascine, terrifie et hante la narratrice. Changeantes, belles et capricieuses, les femmes en vert polymorphes apparaissent et disparaissent sans cesse de la vie de la narratrice pour qui elles sont la mère, l'ex-amie devenue belle-mère, ou encore la maîtresse d'école - celle qui, pour un certain temps, tient la place de la mère. Mais elles sont aussi des revenantes, fantômes de femmes suicidées: Katia Depetiteville et la femme d'Ivan. Toutes ces femmes viennent habiter les photos de Julie Ganzin et sont habitées par elles aussi bien que par les portraits anciens - eux-mêmes répétés dans des panneaux de montage. Phrases et photos se heurtent, se répètent et finissent par renvoyer les unes aux autres. Comme la figure de la mère, ces femmes en vert nées de l'imagination de Ndiaye brouillent la frontière entre la fiction et le réel, la vie et la mort, le présent et le passé. Elles sont, à l'instar du dispositif photographique, la trace de ce qui a été et qu'on perçoit toujours. Pendant que l'écrivaine narre ses aventures à travers une succession de portraits de la/sa mère - «[...] femme en vert, intouchable, décevante, métamorphosable à l'infini [...]» (2005: 72) -, elle forme un objet photo-littéraire qui fonctionne à la manière du corps maternel, lieu du vide et du trop-plein, corps intime et étranger.

Ces résonances puissantes naissent du ricochet entre les signes verbaux et visuels. On retrouve le moment d'aveuglement et de décalage que Philippe Dubois décrit dans L'Acte photographique, le moment de la prise du cliché lorsque le voile noir masque la vision (1983: 143-144). Si le temps du portrait photographique implique un décalage pour le photographe, l'écriture de Ndiaye opère un geste 
représente une étagère dans l'atelier de Nan Goldin, sa bibliothèque et les mots qui la hantent (Plath, Charcot, Didi-Huberman, Amado...); la seconde, prise par son père, est un portrait de Barbara «en masque" à l'âge de sept ans. Il faut voir dans ce portrait une métaphore de ce que représentera pour Nan la pratique photographique: le lendemain éternel du suicide. Nature morte, vanitas, tête de mort, le regard de Barbara est caché derrière un loup noir qui coule sur son visage, le long de son nez, en un mouvement léger qui déplace les trous et tranche les yeux. Le regard de l'enfant est aveugle, à la fois présent et fuyant, tranché parce que décadré.

Cadrer, dit Pascal Bonitzer, est un terme de tauromachie: «immobiliser le taureau avant de l'estoquer». Et le décadrage est un cadrage «arbitraire et nomade» sur "une réalité déshabitée de toute signification pleine».

Au centre du tableau, en principe occupé dans la représentation classique par une présence symbolique (l'image des souverains dans le miroir des Ménines, par exemple), il n'y a rien, il ne se passe rien. L'œil habitué (éduqué?) à centrer tout de suite, à aller au centre, ne trouve rien et reflue à la périphérie, où quelque chose palpite encore, sur le point de disparaitre. [...] L'ironie, c'est montrer froidement, dire froidement la cadavérité.

(Bonitzer, 1985: 84)

Tiré vers un autre centre que celui des trous noirs, crypté parce qu'enfoui à côté, le regard de Barbara emporte dans le mystère tout regard qu'on pourrait poser, parlant cette «langue incomparable de la tête de mort», «unissant l'absence totale d'expression (le noir des orbites) à l'expression la plus sauvage (la grimace de la denture)»?. Le regard de Barbara regarde sans qu'on puisse le voir; il montre qu'il regarde sans qu'on puisse voir ce qu'il est en train de regarder. Ce portrait de Barbara est recadré par sa sœur qui, d'une part, le monumentalise - le transforme en légende - et, d'autre part, se l'approprie comme un objet intime pour en faire une image autobiographique, doublement biographique, donc, comme si les deux sœurs, en siamoises, partageaient un même portrait. En reproduisant l'image de Barbara au sein de son semblable, car la femme décrite dans le portrait narratif n'existe déjà plus. Le portrait littéraire est le portrait d'un temps qui précède la narration. Par ailleurs, l'écart entre l'écriture et la photographie permet à Ndiaye de s'emparer des photos, de déjouer ce rapport étroit au réel. Dans la figure de la revenante et dans les portraits de la «collection particulière", par exemple, on retrouve quelque chose de l'aura que Benjamin finit par accorder au portrait photographique: "[...] l'unique apparition du lointain si proche soit-il» (2003: 19). Pourtant, mis en écho, en série, les portraits sont privés d'origine, d'unicité. Cela trouble l'aura, sceau de l'authenticité, et vient donner un visage non pas à des êtres réels mais à des voix, ces voix que Ndiaye inscrit dans son texte.

De par son écriture et son usage du médium photographique, l'écrivaine tente de figurer ce qui est insaisissable, de donner à voir le visage de celle qui lui échappe par sa métamorphose continuelle. Cependant, comme dans les photos de Julie Ganzin, la figure est floue. Elle est reconnaissable mais, en même temps, elle peut être prise pour une autre. Comme dans les images à double volet ou présentées dans des panneaux, le visage de la femme en vert est répété avec une infime différence. Les portraits narratifs débordent du texte pour inonder les photos comme la Garonne, cette rivière essentiellement féminine qui hante le livre de Ndiaye, qu'elle décrit comme débordant de ses rives. Avec une fausse naïveté, l'écrivaine-narratrice demande: «[...] la Garonne est-elle une ... est-elle une femme en vert?» (2005: 94).

\section{Leur existence, ma propre originalité}

Cette question que pose la narratrice est le dernier moment de doute avant qu'on ne soit invité à lire le récit à rebours. C'est une fausse énigme, un clin d'œil.

Le «je» narratrice-écrivaine ne se nomme jamais, mais le paratexte du livre l'associe au titre et fait du livre un autoportrait - on assiste à la représentation de soi à travers le réalisme merveilleux d'une série de 
œuvre, Nan prend le témoin et le relais. Le portrait devient auto/portrait, l'image de Barbara apparaît comme s'il s'agissait du portrait de Nan. Dans cette perspective, non seulement Sœurs, saintes et sibylles est une autobiographie, mais tous les portraits faits par Goldin sont aussi des autoportraits, comme tous les autoportraits sont aussi des portraits, faux jumeaux, perpétuelle mise en écho.

\section{Self-portrait on the bridge}

Qu'il s'agisse des autres ou d'elle-même, le geste de Goldin est double: il est à la fois saisie, consignation, et perte, deuil, échec inévitable de l'approche de l'autre. C'est la raison pour laquelle elle prend un grand nombre d'images. Car si la photographie, pour Nan Goldin, est vérité, elle n'est jamais que vérité partielle à laquelle il faut toujours plus de vérité: «I don't believe in the single portrait. I believe only in the accumulation of portraits as a representation of a person ${ }^{8}$.

Ainsi, Goldin sérialise, et par le fait même «narrativise», racontant l'histoire de ceux qu'elle photographie comme si elle racontait sa propre histoire. Seul le mode narratif peut nous permettre de comprendre, nous dit Susan Sontag (1977), contrairement aux photos qui ne font que montrer. C'est-à-dire que seule une inscription dans le temps peut engendrer l'entendement. Goldin enchaîne les images comme dans un chemin de croix, elle les relie tout en maintenant un écart, une différence et une répétition. En offrant plusieurs facettes d'un individu, la série multiplie les points de vue et signale le geste vain qui consisterait à vouloir s'approprier un être humain. La série photographique, semblable à celle utilisée par August Sander et Walker Evans dans les années 1930, représente un usage de la composition et de la parataxe, de la distance et de la proximité. C'est ainsi que l'aura réapparaît, par la reproduction et l'écho plutôt que par le fantasme d'une singularité: Narcisse penché au-dessus de son étendue d'eau. "Self-portrait on the bridge», autoportrait fait par Goldin en 1998 dans l'enceinte d'un hôpital, montre l'ombre de la photographe sur l'eau dorée et portraits. Ce jeu de bascule (ou glissement) est parallèle aux métamorphoses des femmes en vert. C'est le moment où le regard de la portraitiste se transforme en regard de portraiturée, où elle se penche sur l'eau, comme Narcisse, afin de se voir. Ainsi, on pourrait dire que, dans un premier temps, Ndiaye représente Écho. Elle disparaît, ou s'esquive, prêtant sa voix aux autres femmes en vert pendant qu'elles se démultiplient et se dédoublent dans les figures maternelles du récit. Sous le regard de la narratrice, ces femmes se reflètent les unes dans les autres, comme elles se mirent dans la Garonne et finalement, rétroactivement, dans le miroir de l'appareil photographique. Sous la plume de Ndiaye, leurs voix sont retranscrites à côté de leurs fauxportraits, mais, dans un deuxième temps, Ndiaye occupe la place de Narcisse. L'auteure renverse le rapport présence-absence: le portrait de la femme en vert devient son miroir. Schaeffer explique:

Le portrait ne cesse ainsi de réactiver une dynamique qui est constitutive de notre anthropoïsation, celle qui ne nous fait atteindre à notre identité qu'à travers le regard d'un autre. Lorsqu'on oppose le portrait à l'autoportrait, on oublie souvent que tout portrait est aussi virtuellement l'autoportrait $d u$ portraituré qui s'y reconnaît et pour qui il fait fonction de prothèse visuelle lui permettant de s'assurer de son identité en tant qu'elle lui est réadressée à travers un regard extérieur [...].

Ndiaye pose la question de l'authenticité, question qui intéressait Benjamin dans L'Euvre d'art à l'époque de sa reproductibilité technique: quelle est la différence entre un original et une copie? Où qu'elle aille, Ndiaye trouve son image... De par ces voyages, la narratrice rappelle le divorce entre lieu et existence, emblématique de la condition moderne, que Benjamin associe à la circulation des œuvres d'art et une désacralisation consécutive au fait qu'elles sont faciles à exposer. Cependant, la notion d'unicité existentielle est inutile pour Ndiaye qui réclame une image changeante lui permettant de voir les multiples facettes de son être: femme française d'origine africaine, mère, amie, fille, écrivaine... Au lieu de 
miroitante d'un cours d'eau, alors qu'elle est appuyée à la balustrade d'un pont ${ }^{9}$. L'ombre est un contour vague, une forme humaine indéterminée, que seule la légende permet d'identifier. Goldin offre un autoportrait-qui-n'en-est-pas-un, image d'elle-même anonyme. Mise en échec du narcissisme ${ }^{10}$ et emprunt de la technique employée pour les amis issus du «demimonde " (où la légende permet de singulariser ceux qui habituellement sont stéréotypés), cet autoportrait n'est pas différent des autoportraits où Goldin se met en scène plus directement, visage fardé, embrouillé ou marqué de coups, drogué ou rêveur, seul ou entouré. Si d'aucuns accusent la photographe de narcissisme, comme si elle était éprise d'elle-même ${ }^{11}$, c'est par un geste qui consiste à prendre son visage en rafale, donc à ne jamais en arrêter l'image qu'elle se représente. Le miroir de l'eau ondule, le visage se fait et se défait sans arrêt, comme si, portant le spectre du suicide de Barbara, Nan jouait figurativement avec la mort. Déjouant la fin de Narcisse, elle occupe alors la place d'Écho, rendant sans cesse hommage à une sœur, dont du vivant elle n'avait jamais pris de photos, comme à tous les aimés présents ou disparus, dont elle répète sans cesse les noms - son art ainsi mis au service d'un travail contre l'oubli.

\section{Tell the children it was an accident}

Le 12 avril 1965, à l'heure du dîner familial, des policiers sonnent à la porte d'une maison en banlieue de Washington pour annoncer à des parents que leur fille s'est jetée sous un train: «J'ai entendu ma mère dire aux policiers: "Dites aux enfants que c'était un accident" "(Goldin, 2005 : n.p.).

Les parents avaient donné à leur fille aînée un nom en palindrome. Après sa mort, la petite Nancy, celle que Barbara aimait tant materner, rendue muette par sa disparition, quitte la maison familiale, ampute son prénom et se révèle ainsi doublement un écho. Nan renaîtra en gardienne et faiseuse d'images, déterrant les archives familiales pour contrer «la tyrannie du révisionnisme», cette réécriture de l'histoire effectuée «immédiatement avant qu'elle ne soit écrite» (ibid.). Elle se fait dès lors gardienne des fixer une seule image d'elle-même, elle se met en série et en reflets:

Car je pense à ma mère, à la femme d'Ivan, à ma belle-mère, et je redoute de me considérer moi-même comme un être insensé si toutes ces femmes en vert disparaissent l'une après l'autre, me laissant dans l'impossibilité de prouver leur existence, ma propre originalité. Je me demande alors [...] comment trouver supportable une vie dénuée de femmes en vert découpant en arrière-plan leur silhouette équivoque. Il me faut, pour traverser calmement ces moments d'hébétude, d'ennui profond, de langueur désemparante, me rappeler qu'elles ornent mes pensées, ma vie souterraine, qu'elles sont là, à la fois êtres réels et figures littéraires sans lesquelles l'âpreté de l'existence me semble racler peau et chair jusqu'à l'os. (Ndiaye, 2005: 77)

Mis en série, le «je», par la rencontre entre texte et photo, s'empare des images recyclées et détournées, créant un effet d'inquiétante étrangeté au lieu du rapport référentiel entre texte et photographie. L'image de soi est troublante et troublée, elle hante le récit par l'entremise des images des autres. Selon Marie-Laure Hurault, la mise en série renforce la fragmentation et le décentrage: "[l]a série refuse, au vu de la dynamique en cours, la position d'un milieu qui aurait une fonction de centre" (2003: 10). Au lieu de dépérir devant l'image unique de soi, Ndiaye se démultiplie dans la série; elle s'énonce dans la résonance entre phrase et photo.

\section{Que l'écriture me rendrait visible et me protégerait}

Autoportrait en vert est ainsi constitué de battements, de face-à-face qui laissent advenir l'image photo-littéraire de l'auteure. Sans être une autobiographie, le texte est autobiographique au sens où l'entend Leigh Gilmore (1994): c'est une représentation de soi à travers les codes contradictoires de la vérité. Si Ndiaye ment dans la mesure où elle dresse un portrait fictif, elle se livre au lecteur-spectateur en tant qu'écrivaine. Dans un entretien, elle dit de l'écriture:

J'espérais qu'elle me sauve de la vie réelle et ordinaire qui me semblait terrifiante. Qu'elle fasse de moi quelqu'un de spécial, d'unique même. J'avais l'impression, enfant, d'être invisible. 
mots, mais aussi surface sur laquelle rebondissent les échos. De fait, Sours, saintes et sibylles non seulement entremêle des photos de famille et des photos prises par Nan Goldin, mais se révèle un creuset de voix, de citations: à côté du récit autobiographique et des légendes photographiques de Goldin, se lisent un certificat de naissance, des extraits des dossiers médicaux, des coupures de journaux, des propos tenus par les psychiatres et les parents, des phrases écrites, prononcées ou transcrites par la sœur suicidée... Renaissent ainsi une époque, un événement, des vies, tous portés par la voix de celle qui les traduit ${ }^{12}$, et qui sont ainsi appelés à rester.

Ce retour en arrière, cette place de médium occupée par Nan Goldin qui fait revivre les autres à travers elle-même, se double d'un mouvement proleptique, une plongée vers l'avant, prophétie digne du livre des sibylles. L'histoire racontée par la photographe est tissée d'allers-retours, elle qui revoit le passé comme annonciateur et traque les oracles. Mais cette recherche, comme Janus, est double: regard derrière et devant soi, prémonition et mise en garde devant l'avenir, transmission d'un savoir. Ici réapparaissent le mythique, le sacré, et avec eux l'aura. Dans le dernier tiers du livre, des photos récentes viennent figurer ce lointain si proche que Benjamin trouvait dans l'admiration des paysages: l'intensité d'un chien-loup, la luminosité de la neige, le spectre de la lune contre le mur d'une chambre. Un avion disparaît dans les nuages, le soleil se couche et une chevelure céleste apparaît; amants, parents et amis sont photographiés en penseurs ou ensommeillés, bientôt emportés par la mort. Reste, à la fin, ce qui doit bien finir: une histoire ratée, des paroles non entendues. La tombe de la sœur, une Nan Goldin de cire figée dans la folie, un père de cire foudroyé par l'éclair: ce qui est arrivé est passé et doit désormais dormir/ce qui est arrivé peut encore se produire, il ne faut pas oublier.

Goldin porte les stigmates de l'histoire ${ }^{13}$, et son missel est à la fois témoignage et leçon, directives concernant le rituel et la transmission. C'est ainsi que son œuvre, exemple éminent de cette désacralisation
J'espérais, sans que cela soit conscient, que l'écriture me rendrait visible et me protégerait en même temps. (Argand, 2001: n.p.)

De par son usage de la photographie, Ndiaye fait écho au Manifeste photobiographique de Gilles Mora et Claude Nori: "[l]a photobiographie étirera le temps de la prise de vue, modifiera notre passé et reconstruira notre vie en une fiction, somme toute véridique, faite d'instants devenus romanesques" (1983: 15). De même, pour revenir au flottement entre portrait et autoportrait, on peut dire que si Ndiaye se rend visible à travers une mise en fiction d'elle-même, ses ouvrages de fiction la cachent autant qu'ils la montrent. En même temps qu'elle se représente, Ndiaye réclame une place dans la succession littéraire, car son livre est traversé de renvois aux œuvres d'autrui aussi bien qu'aux divers ouvrages qui composent la sienne ${ }^{14}$.

Les renvois, résonances et reflets qui traversent Autoportrait en vert sont le lieu de l'intersection des voix et des visages. Ndiaye prête sa voix aux figures des portraits qui parsèment son texte. Dans la dialectique entre texte et image, elle fait cadeau à Écho d'un visage et à Narcisse d'une voix. Par le biais des portraits, elle entraîne le lecteur-spectateur dans un jeu de regards qui met en doute la fixité de l'identité. Comme toujours, elle travaille l'étrangeté, manifeste dans sa résistance à fixer son image ou celle de l'autre. Portant l'attention sur les moments de métamorphose, elle se penche sur l'entre-deux de l'identité, mouvement qui rappelle l'invitation de Georges Didi-Huberman:

Il y a, il n'y a qu'à s'inquiéter de l'entre. Il n'y a qu'à tenter de dialectiser, c'est-à-dire tenter de penser l'oscillation contradictoire dans son mouvement de diastole et de systole [...] à partir de son point central, qui est son point d'inquiétude, de suspens, d'entredeux. Il faut tenter de revenir au point d'inversion et de convertibilité, au moteur dialectique de toutes les oppositions. C'est le moment où ce que nous voyons commence juste d'être atteint par ce qui nous regarde - un moment qui n'impose ni le trop-plein de sens (que glorifie la croyance), ni l'absence cynique de sens (que glorifie la tautologie). C'est le moment où s'ouvre l'antre creusé par ce qui nous regarde dans ce que nous voyons.

(1992: 51-52) 
propre à l'art contemporain, comporte néanmoins une dimension sacrée: cette aura qui confère aux choses qu'on regarde le pouvoir de lever, sur nous, les yeux. Il ne s'agit donc pas, ici, d'un théâtre digne de Narcisse où on le verrait tomber sur lui-même, mais bien du théâtre de l'ignorée et de l'oubliée, Écho, sœur sibylline, visage qu'on ne voit pas mais dont les paroles se reflètent en nous, visage qui apparaît grâce à sa voix.
C'est le moment où Narcisse voit Écho, où Écho prête sa voix à Narcisse penché sur l'eau qui coule entre les deux rives de la Garonne, cette rivière «d'essence féminine» (Ndiaye, 2005: 8).

La photographie est à la fois émanation directe du réel (par le procédé même qui fait surgir l'image - la lumière sur l'objet) et déplacement par le procédé de fictionnalisation: non seulement la présence du regard singulier derrière l'objectif met en péril toute reproduction du réel comme réel, mais le temps photographique est tel qu'il est impossible de concevoir la photo comme une empreinte authentique - le délai entre le présent et la photo, mais aussi la coupure inévitable que provoque la fermeture de l'obturateur et le fait que, pendant un instant, on ne voit pas la scène en train d'être photographiée. Ainsi, pour reprendre la définition proposée par Isabelle de Maison Rouge de l'autofiction: «l'autofiction, c'est transposer sa vie dans le champ de l'impossible, celui de l'écriture ou de la mise en images, comme un lieu qui n'aura jamais lieu» (2004: 21; nous soulignons). Sœurs, saintes et sibylles et Autoportrait en vert sont-ils représentatifs de ce travail de l'intime et de l'autofiction qui, selon Isabelle de Maison Rouge, fait partie intégrante de l'art contemporain? Ces œuvres sont-elles représentatives d'une pratique intermédiale par laquelle les femmes artistes contemporaines explorent l'intime? L'usage conjoint du texte et de l'image dans l'autobiographie est-il un des moyens mis en ouvre par les artistes et écrivaines pour inquiéter la représentation de soi et ainsi doter l'autoportrait d'une aura?

La surface dans laquelle elles se mirent est brouillée, l'image qui apparaît est mouvante et démultipliée, comme si Écho plutôt que Narcisse était penchée sur l'eau. Est-il possible de mettre en image une voix? semblent demander Goldin et Ndiaye. Comment une voix peut-elle apparaître en image? Quel est le visage de l'écho? 


\section{N O TES}

1. Sœurs, saintes et sibylles est la publication, sous forme de livre, d'une installation de son et d'images - diaporamas, séquences vidéos, voix off, musique - à la Chapelle Saint-Louis de la Salpêtrière à l'automne 2004 à Paris. Perché au-dessus de la scène, sur un balcon-passerelle, le public se trouvait devant trois écrans, sur lesquels étaient projetées des diapositives, et surplombait une femme de cire couchée dans un lit, au niveau du sol, comme dans une scène des années 1950-1960 aux ÉtatsUnis. L'installation tire une partie de son sens du lieu choisi, la Salpêtrière évoquant Charcot, l'enfermement des femmes et l'invention de l'hystérie.

2. A. Rouillé parle de sécularisation dans le contexte de l'art contemporain, description qui correspond au travail de Goldin : «La sécularisation n'a rien d'une dissolution de l'art dans le monde, ni d'une abolition de la distance qui les sépare. Il s'agit plutôt d'un déplacement de leurs frontières, d'une reconfiguration des passages, d'une redistribution des seuils et des clôtures [...] la sécularisation se distingue par la place qu'y occupe le matériau-photographie; par des orientations spécifiques - des grands aux petits récits, de la profondeur à la surface, du visible à l'imprésentable, de la haute à la basse culture, des corps aux flux corporels - soutenues, reprises et amplifiées par l'artphotographie» (2005: 524-526).

3. Les critiques ont remarqué la présence du sacré dans l'œuvre de Goldin, manifeste dans les nombreux lits annonciateurs, les chambres d'hôpital ou d'hôtel comme dans les cellules de couvent, les scènes aquatiques comme autant de baptêmes, les ex-voto, l'hagiographie des proches, les cierges de Fatima, etc. (voir Liebmann, 2002 : 120).

4. "C'est un lieu commun de dire que le photographe est, par sa nature même, un voyeur, celui qui n'a pas été invité à la fête. Mais je ne viens pas à la fête sans avoir été invitée; ceci est ma fête. Ceci est ma famille, mon histoire"(Holborn, 1986: 42; notre traduction).

5. Cette expression renvoie à l'exposition "The Family of Man" organisée par Edward Steichen dans les années 1950.

6. On se rappelle un film (pour la BBC) et une exposition antérieure (Whitney Museum), auxquels Goldin a donné le titre «I'll be your mirror». Comme le souligne Lebovici, au pronom anglais est conféré la tâche de gérer la distance. Le "you " signifiant à la fois «tu " et "vous ", il nous engage à penser intimement et politiquement.

7. Benjamin cité par Didi-Huberman (1992: 113).

8. "Je ne crois pas dans la possibilité d'un seul portrait. Je crois seulement en l'accumulation de portraits en tant que représentation d'une personne" (Goldin, 1996: 454 ; notre traduction).

9. Il faut noter la fréquence, dans la production de Goldin, de baigneurs et de baigneuses.

10. Voir la critique que fait É. Roudinesco du narcissisme contemporain (2001).

11. Commentaire fréquent sur les productions féminines contemporaines, qui fait l'impasse sur le travail de désubjectivation qui les sous-tend.

12. Il faut aussi noter la dimension bilingue de cet ouvrage, où chaque passage en français est traduit en anglais, des divergences remarquables distinguent les deux versions.

13. On revoit l'autoportrait de Goldin «Nurses bandaging wounds. The Priory Hospital, London, 2003 ", qui montre des brûlures sur l'avantbras, brûlures de cigarettes qu'elle s'est elle-même faites.

14. On donne à titre d'exemple la comparaison entre les sœurs Papin et les sœurs d'Autoportrait en vert. Cette référence renvoie à la pièce Les Bonnes (1978) de J. Genet qui était une source de la pièce Hilda (1999) de Ndiaye. Cette pièce a été publiée chez Minuit et a été produite au Théâtre du Rond Point en 2006. Voir le dossier de presse du théâtre.

\section{RÉFÉREN CES BIBLIO G RAPHIQ U ES}

ARGAND, C. [2001]: "Marie Ndiaye: Entretien ", Lire, avril. En ligne: http://www.lire.fr/entretien.asp/idC=36945/idTC=4/idR=201/idG (page consultée le 10 janvier 2007).

BENJAMIN, W. [(1939) 2003] : L'Euvre d'art à l'époque de sa reproductibilité technique, Paris, Éd. Allia.

Bonitzer, P. [1985]: Peinture et Cinéma. Décadrages, Paris, Éd. de l'Étoile.

COHEN, J. (dir.) [2006]: L'Art et le politique interloqués, Paris,

L'Harmattan.

Didi-HubERMAN, G. [1992]: Ce que nous voyons, ce qui nous regarde,

Paris, Minuit, coll. "Critique».

DuBOIS, P. [1983]: L'Acte photographique et autres essais, Paris et

Bruxelles, Fernand Nathan et Éd. Labor;

[1991]: «Palimpsestes ou la photographie comme appareil psychique», Cahiers figures: Mythologies du photographe, no 8, 103-128. GiLmORE, L. [1994] : Autobiographics : a Feminist Theory of Women's Selfrepresentation, Ithaca, Cornell University Press, coll. «Reading Women Writing».

Goldin, N. [1996] : I'll Be Your Mirror, New York, Whitney Museum of American Art;

- [2005]: Sœurs, saintes et sibylles, Paris, Éd. du Regard.

Holborn, M. [1986]: "Nan Goldin's Ballad of Sexual Dependancy", Aperture 103, 38-47.

Hurault, M.-L. [2003] : "La mise en série", dans F. Brunet et alii (dir.), Effets de cadres: de la limite en art, Saint-Denis, Presses universitaires de Vincennes, 9-18.

LaCoue-Labarthe, P. [1979]: Portrait de l'artiste en général, Paris,

Christian Bourgois, coll. "Première Livraison".

LEBOVICI, E. [1999]: “On the edges of the image”, Parkett, no 57, 68-72.

LIEBMANN, L. [2002]: "Goldin's Years : Nan Goldin in Retrospect”, Artforum International, vol. 41, no 2, 118-123.

MAISON Rouge, I. de [2004]: Mythologies personnelles. L'art contemporain et l'intime, Paris, Éd. Scala.

MORA, G. et C. NORI [1983]: L'Été dernier. Manifeste photobiographique,

Paris, Éd. de l'Étoile, coll. "Écrits sur l'image».

NDIAYE, M. [1999]: En famille, Paris, Minuit;

[2001]: Rosie Carpe, Paris, Minuit;

[2005]: Autoportrait en vert, Paris, Mercure de France, coll.

"Traits et portraits".

ROUDINESCO, É. [2001] : L'Analyse, l'Archive, Paris, Bibliothèque

nationale de France.

ROUILLÉ, A. [2005]: La Photographie, Paris, Gallimard.

SCHAEFFER, J.-M. [1997]: «Du portrait photographique», dans

P. Arbaizar (dir.), Portraits singulier-pluriel 1980-1990; le photographe et son modèle, catalogue d'exposition (14 octobre au 18 janvier 1997), Paris,

F. Hazan et Bibliothèque nationale de France, 9-25.

SONTAG, S. [1977]: On Photography, New York, Farrar, Straus \& Giroux. THÉÂTRE Du Rond POINT [2006] : Dossier de Presse. Hilda., dans N. de Sultan (dir.). En ligne: http://www.theatredurondpoint.fr/pdf/ dp_30305.pdf (page consultée le 10 janvier 2007). 